\title{
On Vector-Valued Generalized Lorentz Difference Sequence Space
}

\author{
Birsen Sağır and Oğuz Oğur \\ Department of Mathematics, Art and Science Faculty, Ondokuz Mayis University, Kurupelit Campus, 55139 Samsun, Turkey \\ Correspondence should be addressed to Oğuz Oğur; oguz.ogur@omu.edu.tr
}

Received 19 June 2014; Accepted 15 September 2014; Published 21 September 2014

Academic Editor: Lingju Kong

Copyright (C) 2014 B. Sağır and O. Oğur. This is an open access article distributed under the Creative Commons Attribution License, which permits unrestricted use, distribution, and reproduction in any medium, provided the original work is properly cited.

We introduce generalized Lorentz difference sequence spaces $d(v, \Delta, p)$. Also we study some topologic properties of this space and obtain some inclusion relations.

\section{Introduction}

Throughout this work, $\mathbb{N}, \mathbb{R}$, and $\mathbb{C}$ denote the set of positive integers, real numbers, and complex numbers, respectively.

The notion of difference sequence space was introduced by Kizmaz in [1] in 1981 as follows:

$$
X(\Delta)=\left\{x=\left\{x_{k}\right\} \in w:\left(\Delta x_{k}\right) \in X\right\}
$$

for $X=\ell_{\infty}, c, c_{0}$, where $\Delta x_{k}=x_{k}-x_{k+1}$ for all $k \in \mathbb{N}$. Et and Çolak in [2] defined the sequence space

$$
X\left(\Delta^{m}\right)=\left\{x=\left\{x_{k}\right\} \in w:\left(\Delta^{m} x_{k}\right) \in X\right\}
$$

for $X=\ell_{\infty}, c, c_{0}$, where $m \in \mathbb{N}, \Delta^{0} x_{k}=\left\{x_{k}\right\}, \Delta x_{k}=x_{k}-x_{k+1}$, $\Delta^{m} x_{k}=\Delta^{m-1} x_{k}-\Delta^{m-1} x_{k+1}=\sum_{v=1}^{m}(-1)^{v}\left(\begin{array}{c}m \\ v\end{array}\right) x_{k+v}$ for all $k \epsilon$ $\mathbb{N}$, and showed that this space is a Banach space with norm

$$
\|x\|_{\Delta}=\sum_{i=1}^{m}\left|x_{i}\right|+\left\|\Delta^{m} x\right\|_{\infty}
$$

Subsequently difference sequence spaces has been discussed in Ahmad and Mursaleen [3], Malkowsky and Parashar [4], Et and Basarir [5], and others.
Let $(E,\|\cdot\|)$ be a Banach space. The Lorentz sequence space $l(p, q, E)\left(\right.$ or $\left.l_{p, q}(E)\right)$ for $1 \leq p, q \leq \infty$ is the collection of all sequences $\left\{a_{i}\right\} \in c_{0}(E)$ such that

$$
\left\|\left\{a_{i}\right\}\right\|_{p, q}=\left\{\begin{array}{c}
\left(\sum_{i=1}^{\infty} i^{q / p-1}\left\|a_{\phi(i)}\right\|^{q}\right)^{1 / q} \\
\text { for } 1 \leq p \leq \infty, \quad 1 \leq q<\infty \\
\operatorname{supi}_{i}^{1 / p}\left\|a_{\phi(i)}\right\| \\
\text { for } 1 \leq p<\infty, \quad q=\infty
\end{array}\right.
$$

is finite, where $\left\{\left\|a_{\phi(i)}\right\|\right\}$ is nonincreasing rearrangement of $\left\{\left\|a_{i}\right\|\right\}$ (we can interpret that the decreasing rearrangement $\left\{\left\|a_{\phi(i)}\right\|\right\}$ is obtained by rearranging $\left\{\left\|a_{i}\right\|\right\}$ in decreasing order). This space was introduced by Miyazaki in [6] and examined comprehensively by Kato in [7].

A weight sequence $v=\{v(i)\}$ is a positive decreasing sequence such that $v(1)=1, \lim _{i \rightarrow \infty} v(i)=0$ and $\lim _{i \rightarrow \infty} V(i)=\infty$, where $V(i)=\sum_{n=1}^{i} v(n)$ for every $i \epsilon$ $\mathbb{N}$. Popa [8] defined the generalized Lorentz sequence space $d(v, p)$ for $0<p<\infty$ as follows:

$$
\begin{aligned}
d(v, p)=\{x & =\left\{x_{i}\right\} \in w:\|x\|_{v, p} \\
& \left.=\sup _{\pi}\left(\sum_{i=1}^{\infty}\left|x_{\pi(i)}\right|^{p} v(i)\right)^{1 / p}<\infty\right\},
\end{aligned}
$$


where $\pi$ ranges over all permutations of the positive integers and $v=\{v(i)\}$ is a weight sequence. It is known that $d(v, p) \subset$ $c_{0}$ and hence for each $x \in d(v, p)$ there exists a nonincreasing rearrangement $\left\{x^{*}\right\}=\left\{x_{i}^{*}\right\}$ of $x$ and

$$
\|x\|_{v, p}=\left(\sum_{n=1}^{\infty}\left|x_{i}^{*}\right|^{p} v(i)\right)^{1 / p}
$$

(see $[8,9])$.

Let $(X,\|\cdot\|)$ be a Banach space and let $v=\{v(n)\}$ be a weight sequence. We introduce the vector-valued generalized Lorentz difference sequence space $d(v, \Delta, p)$ for $0<p<$ $\infty$. The space $d(v, \Delta, p)$ is the collection of all $X$-valued 0 sequences $\left\{x_{n}\right\}\left(\left\{x_{n}\right\} \in c_{0}\{X\}\right)$ such that

$$
\left(\sum_{n=1}^{\infty}\left[\left\|\Delta x_{\phi(n)}\right\|\right]^{p} v(n)\right)^{1 / p}
$$

is finite, where $\left\{\left\|\Delta x_{\phi(n)}\right\|\right\}$ is nonincreasing rearrangement of $\left\{\left\|\Delta x_{n}\right\|\right\}$ and $\Delta x_{\phi(n)}=x_{\phi(k)}-x_{\phi(k+1)}$ for all $k \in \mathbb{N}$.

We will need the following lemmas.

Lemma 1 (see [10]). Let $\left\{c_{i}^{*}\right\}$ and $\left\{{ }^{*} c_{i}\right\}$ be the nonincreasing and nondecreasing rearrangements of a finite sequence $\left\{c_{i}\right\}_{1 \leq i \leq n}$ of positive numbers, respectively. Then for two sequences $\left\{a_{i}\right\}_{1 \leq i \leq n}$ and $\left\{b_{i}\right\}_{1 \leq i \leq n}$ of positive numbers we have

$$
\sum_{i} a_{i}^{*} \cdot{ }^{*} b_{i} \leq \sum_{i} a_{i} \cdot b_{i} \leq \sum_{i} a_{i}^{*} \cdot b_{i}^{*}
$$

Lemma 2 (see [7]). Let $\left\{x_{i}^{(\mu)}\right\}$ be an $X$-valued double sequence such that $\lim _{i \rightarrow \infty} x_{i}^{(\mu)}=0$ for each $\mu \in \mathbb{N}$ and let $\left\{x_{i}\right\}$ be an $X$-valued sequence such that $\lim _{\mu \rightarrow \infty} x_{i}^{(\mu)}=x_{i}$ (uniformly in i). Then $\lim _{i \rightarrow \infty} x_{i}=0$ and for each $i \in \mathbb{N}$

$$
\left\|x_{\phi(i)}\right\| \leq \lim _{\mu \rightarrow \infty}\left\|x_{\phi_{\mu}(i)}^{(\mu)}\right\|
$$

where $\left\{\left\|x_{\phi(i)}\right\|\right\}$ and $\left\{\left\|x_{\phi_{\mu}(i)}^{(\mu)}\right\|\right\}_{i}$ are the nonincreasing rearrangements of $\left\{\left\|x_{i}\right\|\right\}$ and $\left\{\left\|x_{i}^{(\mu)}\right\|\right\}_{i}$, respectively.

\section{Main Results}

Theorem 3. The space $d(v, \Delta, p)$ for $0<p<\infty$ is a linear space over the field $K=\mathbb{R}$ or $\mathbb{C}$.

Proof. Let $x, y \in d(v, \Delta, p)$ and let $\left\{\left\|\Delta x_{\phi(n)}\right\|\right\},\left\{\left\|\Delta y_{\eta(n)}\right\|\right\}$ and $\left\{\left\|\Delta x_{\psi(n)}+\Delta y_{\psi(n)}\right\|\right\}$ be the nonincreasing rearrangements of the sequences $\left\{\left\|\Delta x_{n}\right\|\right\},\left\{\left\|\Delta y_{n}\right\|\right\}$ and $\left\{\left\|\Delta x_{n}+\Delta y_{n}\right\|\right\}$, respectively. Since $v$ is nonincreasing, by Lemma 1 we have

$$
\begin{aligned}
& \sum_{n=1}^{\infty}\left\|\Delta x_{\psi(n)}+\Delta y_{\psi(n)}\right\|^{p} v(n) \\
& \quad \leq D \sum_{n=1}^{\infty}\left(\left\|\Delta x_{\psi(n)}\right\|^{p} v(n)+\left\|\Delta y_{\psi(n)}\right\|^{p} v(n)\right) \\
& \quad \leq D\left\{\sum_{n=1}^{\infty}\left\|\Delta x_{\phi(n)}\right\|^{p} v(n)+\sum_{n=1}^{\infty}\left\|\Delta y_{\eta(n)}\right\|^{p} v(n)\right\} \\
& \quad<\infty,
\end{aligned}
$$

where $D=\max \left\{1,2^{p-1}\right\}$. Let $\alpha \in K$. Hence we get

$$
\begin{aligned}
\sum_{n=1}^{\infty}\left\|\Delta(\alpha x)_{\phi(n)}\right\|^{p} v(n) & =\sum_{n=1}^{\infty}\left\|\alpha \Delta x_{\phi(n)}\right\|^{p} v(n) \\
& =|\alpha|^{p} \sum_{n=1}^{\infty}\left\|\Delta x_{\phi(n)}\right\|^{p} v(n) \\
& <\infty .
\end{aligned}
$$

This shows that $x+y \in d(v, \Delta, p), \alpha x \in d(v, \Delta, p)$ and so $d(v, \Delta, p)$ is a linear space.

Theorem 4. The space $d(v, \Delta, p)$ for $1 \leq p<\infty$ is normed space with the norm

$$
\|x\|_{v, \Delta, p}=\left\|x_{\phi(1)}\right\|+\left(\sum_{n=1}^{\infty}\left\|\Delta x_{\phi(n)}\right\|^{p} v(n)\right)^{1 / p}
$$

where $\left\{\left\|\Delta x_{\phi(n)}\right\|\right\}$ denotes the nonincreasing rearrangements of $\left\{\left\|\Delta x_{n}\right\|\right\}$.

Proof. It is clear that $\|0\|_{v, \Delta, p}=0$. Let $\|x\|_{v, \Delta, p}=0$. Then we have $x_{\phi(1)}=0$ and $\Delta x_{\phi(k)}=x_{\phi(k)}-x_{\phi(k+1)}=0$ for all $k \in \mathbb{N}$. Hence we get $x=0$.

Let $x, y \in d(v, \Delta, p)$. Since weight sequence $v$ is decreasing, by Lemma 1 we have

$$
\begin{aligned}
& \|x+y\|_{v, \Delta, p} \\
& =\left\|x_{\psi(1)}+y_{\psi(1)}\right\|+\left(\sum_{n=1}^{\infty}\left\|\Delta x_{\psi(n)}+\Delta y_{\psi(n)}\right\|^{p} v(n)\right)^{1 / p} \\
& \leq\left\|x_{\psi(1)}\right\|+\left\|y_{\psi(1)}\right\|+\left(\sum_{n=1}^{\infty}\left\|\Delta x_{\psi(n)}\right\|^{p} v(n)\right)^{1 / p} \\
& \quad+\left(\sum_{n=1}^{\infty}\left\|\Delta y_{\psi(n)}\right\|^{p} v(n)\right)^{1 / p}
\end{aligned}
$$




$$
\begin{aligned}
\leq & \left\|x_{\phi(1)}\right\|+\left\|y_{\eta(1)}\right\|+\left(\sum_{n=1}^{\infty}\left\|\Delta x_{\phi(n)}\right\|^{p} v(n)\right)^{1 / p} \\
& +\left(\sum_{n=1}^{\infty}\left\|\Delta y_{\eta(n)}\right\|^{p} v(n)\right)^{1 / p} \\
= & \|x\|_{v, \Delta, p}+\|y\|_{v, \Delta, p}
\end{aligned}
$$

where $\left\{\left\|\Delta x_{\phi(n)}\right\|\right\},\left\{\left\|\Delta y_{\eta(n)}\right\|\right\}$ and $\left\{\left\|\Delta x_{\psi(n)}+\Delta y_{\psi(n)}\right\|\right\}$ denote the nonincreasing rearrangements of $\left\{\left\|\Delta x_{n}\right\|\right\},\left\{\left\|\Delta y_{n}\right\|\right\}$ and $\left\{\left\|\Delta x_{n}+\Delta y_{n}\right\|\right\}$, respectively.

Let $\lambda$ be an element in $K$ and let $x$ be a vector in $d(v, \Delta, p)$. Hence we have

$$
\begin{aligned}
\|\lambda x\|_{v, \Delta, p} & =\left\|(\lambda x)_{\phi(1)}\right\|+\left(\sum_{n=1}^{\infty}\left\|\Delta(\lambda x)_{\phi(n)}\right\|^{p} v(n)\right)^{1 / p} \\
& =|\lambda|\left\|x_{\phi(1)}\right\|+|\lambda|\left(\sum_{n=1}^{\infty}\left\|\Delta x_{\phi(n)}\right\|^{p} v(n)\right)^{1 / p} \\
& =|\lambda|\|x\|_{v, \Delta, p} .
\end{aligned}
$$

Theorem 5. The space $d(v, \Delta, p)$ for $1 \leq p<\infty$ is complete with respect to its norm.

Proof. Let $\left\{x^{(s)}\right\}$ be an arbitrary Cauchy sequence in $d(v, \Delta, p)$ with $x^{(s)}=\left\{x_{n}^{(s)}\right\}_{n=1}^{\infty}$ for all $s \in \mathbb{N}$. Then we have

$$
\lim _{s, t \rightarrow \infty}\left\|x^{(s)}-x^{(t)}\right\|_{v, \Delta, p}=0 .
$$

Hence we obtain $\lim _{s, t \rightarrow \infty}\left\|\Delta x_{\pi_{s, t}(n)}^{(s)}-\Delta x_{\pi_{s, t}(t)}^{(t)}\right\|=0$ for each $n \in \mathbb{N}$ and so $\left\{x_{n}^{(s)}\right\}$, for a fixed $n \in \mathbb{N}$, is a Cauchy sequence in $X$.

Then, there exists $x_{n} \in X$ such that $x_{n}^{(s)} \rightarrow x_{n}$ as $s \rightarrow \infty$. Let $x=\left\{x_{n}\right\}$. Since $\lim _{n \rightarrow \infty} x_{n}^{(s)}=0$ for each $s \in \mathbb{N}$, by Lemma 2 we have $\lim _{n \rightarrow \infty} x_{n}=0$. Therefore we can choose the nonincreasing rearrangement $\left\{\left\|\Delta x_{\pi_{t}(n)}-\Delta x_{\pi_{t}(n)}^{(t)}\right\|\right\}_{n}$ of $\left\{\left\|\Delta x_{n}-\Delta x_{n}^{(t)}\right\|\right\}_{n}$. Also, for an arbitrary $\varepsilon>0$ there exists $N \in \mathbb{N}$ such that

$$
\begin{aligned}
& \left\|x_{\pi_{s, t}(1)}^{(s)}-x_{\pi_{s, t}(1)}^{(t)}\right\| \\
& \quad+\left(\sum_{n=1}^{\infty}\left\|\Delta x_{\pi_{s, t}^{(s)}}^{(s)}-\Delta x_{\pi_{s, t}(n)}^{(t)}\right\|^{p} v(n)\right)^{1 / p}<\varepsilon
\end{aligned}
$$

for $s, t>N$. Hence we get

$$
\begin{aligned}
& \left\|x_{\pi_{s, t}(1)}^{(s)}-x_{\pi_{s, t}(1)}^{(t)}\right\|<\varepsilon, \\
& \sum_{n=1}^{\infty}\left\|\Delta x_{\pi_{s, t}(n)}^{(s)}-\Delta x_{\pi_{s, t}(n)}^{(t)}\right\|^{p} v(n)<\varepsilon^{p}
\end{aligned}
$$

for $s, t>N$. Let $t$ be an arbitrary positive integer with $t>N$ and fixed. If we put

$$
\Delta y_{n}^{(s)}=\Delta x_{n}^{(s)}-\Delta x_{n}^{(t)}, \quad \Delta y_{n}=\Delta x_{n}-\Delta x_{n}^{(t)},
$$

then we have

$$
\begin{aligned}
& \lim _{n \rightarrow \infty} \Delta y_{n}^{(s)}=0 \quad \text { for each } s \in \mathbb{N}, \\
& \lim _{s \rightarrow \infty} \Delta y_{n}^{(s)}=\Delta y_{n} \quad(\text { uniformly in } n) .
\end{aligned}
$$

Thus by Lemma 2 we get

$$
\left\|\Delta y_{\phi(n)}\right\| \leq \lim _{s \rightarrow \infty}\left\|\Delta y_{\phi_{s}(n)}^{(s)}\right\|
$$

for each $n \in \mathbb{N}$; that is,

$$
\left\|\Delta x_{\pi_{t}(n)}-\Delta x_{\pi_{t}(n)}^{(t)}\right\| \leq \lim _{s \rightarrow \infty}\left\|\Delta x_{\pi_{s, t}(n)}^{(s)}-\Delta x_{\pi_{s, t}(t)}^{(t)}\right\|
$$

for each $n \in \mathbb{N}$. Hence, by (17), (21) we get

$$
\begin{aligned}
& \left\|x-x^{(t)}\right\|_{v, \Delta, p} \\
& =\left\|x_{\pi_{t}(1)}-x_{\pi_{t}(1)}^{(t)}\right\| \\
& +\left(\sum_{n=1}^{\infty}\left[\left\|\Delta x_{\pi_{t}(n)}-\Delta x_{\pi_{t}(n)}^{(t)}\right\|\right]^{p} v(n)\right)^{1 / p} \\
& \leq\left\|x_{\pi_{t}(1)}-x_{\pi_{t}(1)}^{(t)}\right\| \\
& +\left(\sum_{n=1}^{\infty}\left(\lim _{s \rightarrow \infty}\left\|\Delta x_{\pi_{s, t}(n)}^{(s)}-\Delta x_{\pi_{s, t}(n)}^{(t)}\right\|\right)^{p} v(n)\right)^{1 / p} \\
& =\left\|x_{\pi_{t}(1)}-x_{\pi_{t}(1)}^{(t)}\right\| \\
& +\lim _{s \rightarrow \infty}\left(\sum_{n=1}^{\infty}\left(\left\|\Delta x_{\pi_{s, t}^{(s)}(n)}-\Delta x_{\pi_{s, t}(n)}^{(t)}\right\|\right)^{p} v(n)\right)^{1 / p} \\
& <2 \varepsilon \text {. }
\end{aligned}
$$

Also, since $d(v, \Delta, p)$ is a linear space we have $\left\{x_{n}\right\}=\left\{x_{n}-\right.$ $\left.x_{n}^{(N)}\right\}+\left\{x_{n}^{(N)}\right\} \in d(v, \Delta, p)$. Hence the space $d(v, \Delta, p)$ is complete with respect to its norm.

Theorem 6. Let $0<p<\infty$. Then, the inclusion $d(v, p) \subset$ $d(v, \Delta, p)$ holds.

Proof. Let $x \in d(v, p)$. Then we have

$$
\left(\sum_{n=1}^{\infty}\left\|x_{\phi(n)}\right\|^{p} v(n)\right)^{1 / p}<\infty,
$$


where $\left\{\left\|x_{\phi(n)}\right\|\right\}$ denotes the nonincreasing rearrangements of $\left\{\left\|x_{n}\right\|\right\}$. Since $v(n)$ is decreasing, by Lemma 1 we get

$$
\begin{aligned}
& \left(\sum_{n=1}^{\infty}\left\|\Delta x_{\psi(n)}\right\|^{p} v(n)\right) \\
& \quad=\sum_{n=1}^{\infty}\left\|x_{\psi(n)}-x_{\psi(n+1)}\right\|^{p} v(n) \\
& \quad \leq K \sum_{n=1}^{\infty}\left(\left\|x_{\psi(n)}\right\|^{p}+\left\|x_{\psi(n+1)}\right\|^{p}\right) v(n) \\
& \quad \leq K\left(\sum_{n=1}^{\infty}\left\|x_{\phi(n)}\right\|^{p} v(n)+\sum_{n=1}^{\infty}\left\|x_{\phi(n+1)}\right\|^{p} v(n)\right) \\
& \quad<\infty,
\end{aligned}
$$

where $\left\{\left\|\Delta x_{\psi(n)}\right\|\right\}$ denotes the nonincreasing rearrangements of $\left\{\left\|\Delta x_{n}\right\|\right\}$ and $K=\max \left\{1,2^{p^{-1}}\right\}$. This completes the proof.

Theorem 7. If $1 \leq p<q<\infty$, then $d(v, \Delta, p) \subset d(v, \Delta, q)$.

Proof. Let $x \in d(v, \Delta, p)$. Since $v(n)$ is decreasing we have

$$
\begin{aligned}
\left(\sum_{n=1}^{\infty}\left\|\Delta x_{\phi(n)}\right\|^{p} v(n)\right)^{1 / p} & \geq\left(\sum_{n=1}^{k}\left\|\Delta x_{\phi(n)}\right\|^{p} v(n)\right)^{1 / p} \\
& \geq\left\|\Delta x_{\phi(k)}\right\|\left(\sum_{n=1}^{k} v(n)\right)^{1 / p} \\
& \geq\left\|\Delta x_{\phi(k)}\right\|(v(k))^{1 / p} k^{1 / p}
\end{aligned}
$$

for every $k \in \mathbb{N}$. Hence we get

$$
\begin{aligned}
& \left\|\Delta x_{\phi(k)}\right\| \\
& \quad \leq(v(k))^{-1 / p} k^{-1 / p}\left(\left\|\Delta x_{\phi(1)}\right\|+\sum_{n=1}^{\infty}\left\|\Delta x_{\phi(n)}\right\|^{p} v(n)\right)^{1 / p} \\
& \quad \leq(v(k))^{-1 / p}\|x\|_{v, \Delta, p}
\end{aligned}
$$

for every $k \in \mathbb{N}$. Thus

$$
\begin{aligned}
& \sum_{n=1}^{\infty}\left\|\Delta x_{\phi(n)}\right\|^{q} v(n) \\
& \quad=\sum_{n=1}^{\infty}\left\|\Delta x_{\phi(n)}\right\|^{q-p}\left\|\Delta x_{\phi(n)}\right\|^{p} v(n) \\
& \quad \leq \sum_{n=1}^{\infty}\left((v(n))^{-1 / p}\|x\|_{v, \Delta, p}\right)^{q-p}\left\|\Delta x_{\phi(n)}\right\|^{p} v(n) \\
& \quad \leq\left(\|x\|_{v, \Delta, p}\right)^{q-p} \sum_{n=1}^{\infty}\left\|\Delta x_{\phi(n)}\right\|^{p} v(n) \\
& \quad<\infty .
\end{aligned}
$$

\section{Conclusion}

If we put $\Delta^{m} x$ instead of $\Delta x$, where $m \in \mathbb{N}$ and $\Delta^{0} x_{k}=$ $\left\{x_{k}\right\}, \Delta x_{k}=x_{k}-x_{k+1}, \Delta^{m} x_{k}=\Delta^{m-1} x_{k}-\Delta^{m-1} x_{k+1}=$ $\sum_{v=1}^{m}(-1)^{v}\left(\begin{array}{c}m \\ v\end{array}\right) x_{k+v}$ for all $k \in \mathbb{N}$ in the definition of $d(v, \Delta, p)$, we obtain generalized Lorentz difference sequence space $d\left(v, \Delta^{m}, p\right)$ of order $m$. It can be shown that the sequence space $d\left(v, \Delta^{m}, p\right)$ is a Banach space with norm

$$
\|x\|_{v, \Delta^{m}, p}=\sum_{n=1}^{m}\left\|x_{\phi(n)}\right\|+\left(\sum_{n=1}^{\infty}\left\|\Delta^{m} x_{\phi(n)}\right\|^{p} v(n)\right)^{1 / p},
$$

where $\left\{\left\|\Delta^{m} x_{\phi(n)}\right\|\right\}$ denotes the nonincreasing rearrangements of $\left\{\left\|\Delta^{m} x_{n}\right\|\right\}$ and properties in this work.

\section{Conflict of Interests}

The authors declare that there is no conflict of interests regarding the publication of this paper.

\section{References}

[1] H. Kizmaz, "On certain sequence spaces," Canadian Mathematical Bulletin, vol. 24, no. 2, pp. 169-176, 1981.

[2] M. Et and R. Çolak, "On some generalized difference sequence spaces," Soochow Journal of Mathematics, vol. 21, no. 4, pp. 377386, 1995.

[3] Z. U. Ahmad and Mursaleen, "Köthe-toeplitz duals of some new sequence spaces and their matrix maps," Publications de l'Institut Mathématique, vol. 42(56), pp. 57-61, 1987.

[4] E. Malkowsky and S. D. Parashar, "Matrix transformations in spaces of bounded and convergent difference sequences of order m, Analysis, vol. 17, no. 1, pp. 87-97, 1997.

[5] M. Et and M. Basarir, "On some new generalized difference sequence spaces," Periodica Mathematica Hungarica, vol. 35, no. 3, pp. 169-175, 1997.

[6] K. Miyazaki, "( $p, q)$-nuclear and $(p, q)$-integral operators," Hiroshima Mathematical Journal, vol. 4, pp. 99-132, 1974.

[7] M. Kato, “On Lorentz spaces $l_{p, q}\{E\}$, , Hiroshima Mathematical Journal, vol. 6, no. 1, pp. 73-93, 1976.

[8] N. Popa, "Basic sequences and subspaces in Lorentz sequence spaces without local convexity," Transactions of the American Mathematical Society, vol. 263, no. 2, pp. 431-456, 1981.

[9] M. Nawrocki and A. Ortynski, "The Mackey topology and complemented subspaces of Lorentz sequence spaces $d(w, p)$ for $0<p<1$," Transactions of the American Mathematical Society, vol. 287, no. 2, pp. 713-722, 1985.

[10] G. H. Hardy, J. E. Littlewood, and G. Pólya, Inequalities, Cambridge University Press, Cambridge, UK, 1967.

This implies that $x \in d(v, \Delta, q)$. 


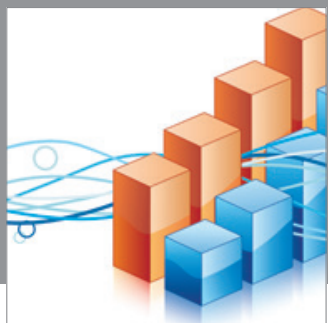

Advances in

Operations Research

mansans

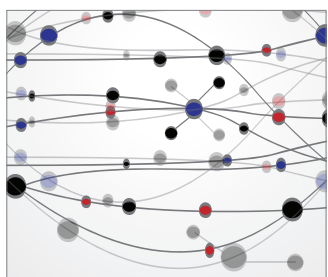

The Scientific World Journal
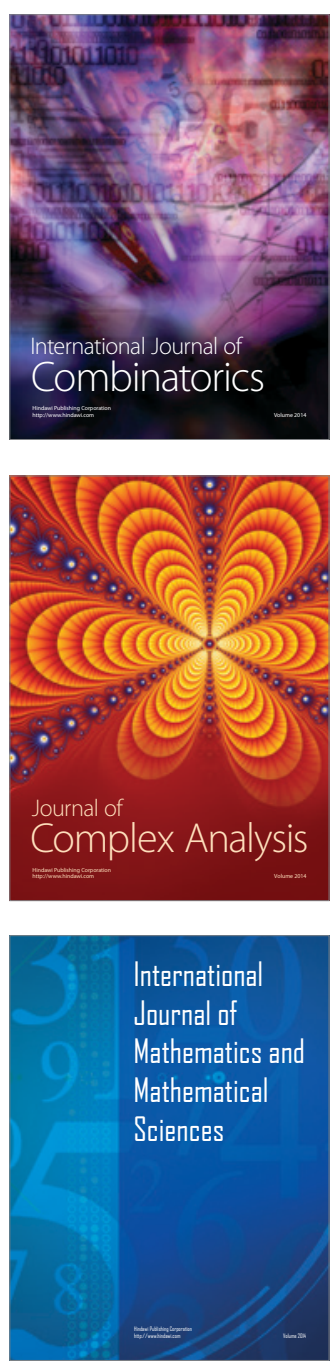
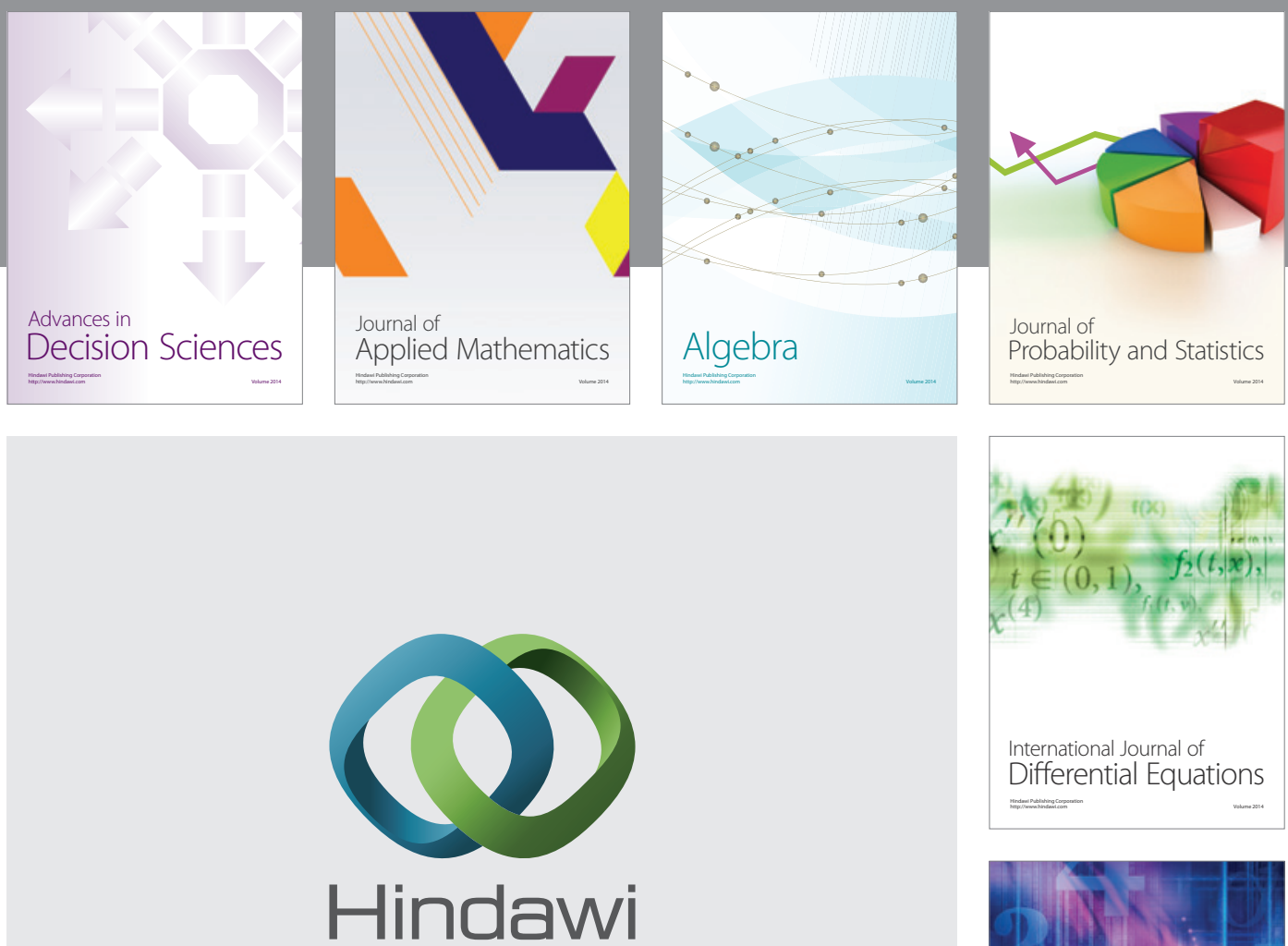

Submit your manuscripts at http://www.hindawi.com
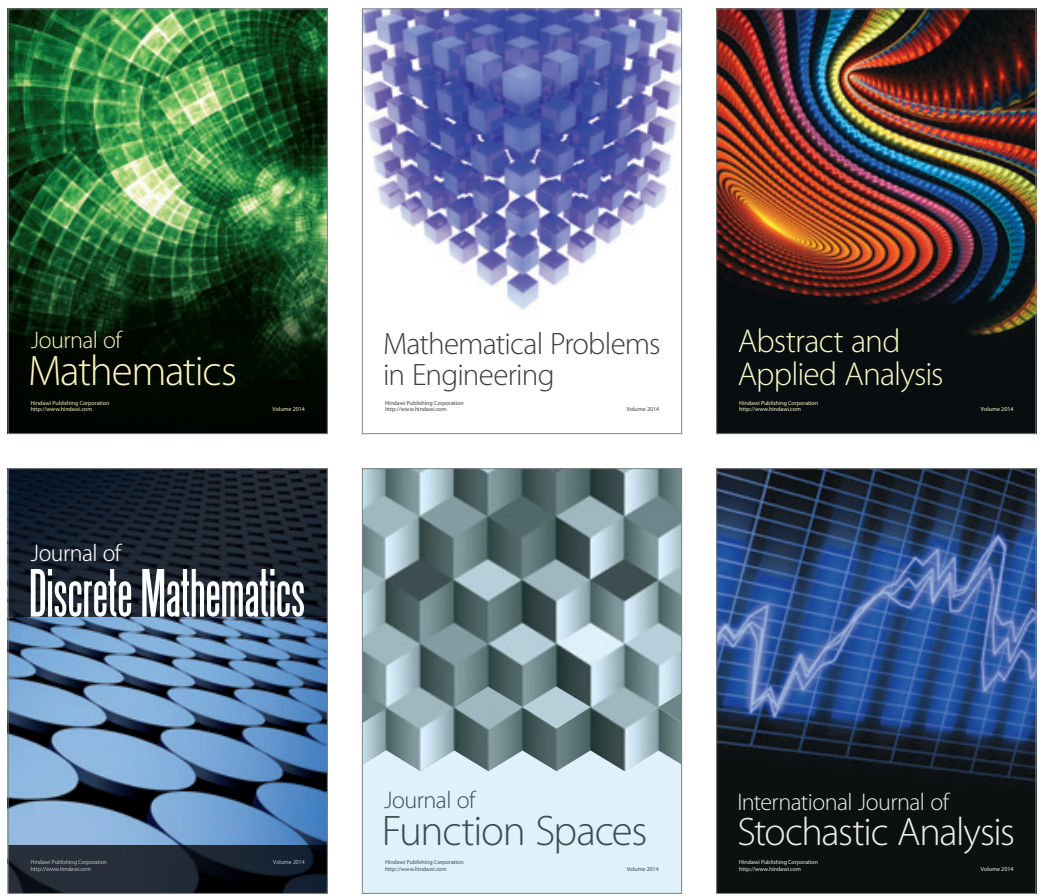

Journal of

Function Spaces

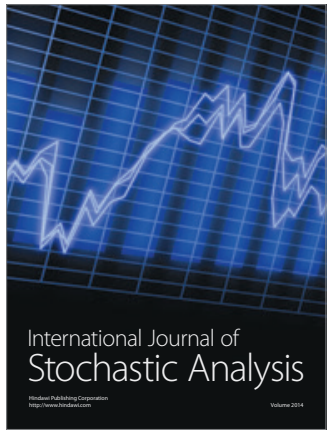

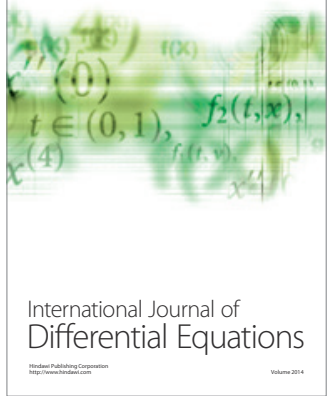
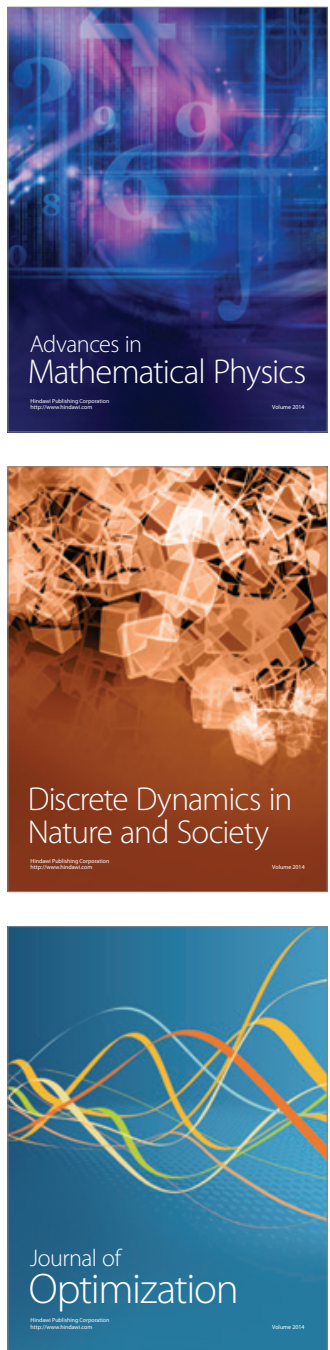